\section{Calidad de vida relacionada con la salud en una muestra de médicos internos residentes que realizan guardias en un Servicio de Urgencias: una perspectiva de género}

\author{
MARÍA FERNÁNDEZ-PRADA ${ }^{1,2}$, JOAQUÍN GONZÁLEZ-CABRERA ${ }^{3}$, \\ FRANCISCO TORRES G. ${ }^{4}$, CONCEPCIÓN IRIBAR-IBABE ${ }^{5,6}$, \\ JOSÉ MARÍA PEINADO ${ }^{5,6}$
}

\section{Gender influence on health related quality of life among resident physicians working in an Emergency Department}

Background: The high emotional burden of physicians working in emergency departments may affect their quality of life perception. Aim: To evaluate health related quality of life among resident physicians performing shifts at an emergency department. Material and Methods: Seventy one physicians aged $26,3 \pm 1,7$ years (47 women), working as residents in an emergency department, answered the short version of the Short-Form Health Survey Questionnaire $\left(S F-36^{\circledR}\right)$. This questionnaire analyses eight domains: physical function, body pain, general health, vitality, social function, emotional role and mental health. Results: Women had a significantly worse perception than a reference population in four dimensions of the SF-36, especially mental health and social functioning. Men had scores similar to the reference population. Among women, vitality is the best predictor of mental health and social functioning. Conclusions: Women working as residents in an emergency department have a worse perception of their quality of life than men performing the same job.

(Rev Med Chile 2014; 142: 193-198) of life.

Key words: Emergencies; Gender identity; Internship and Residency; Quality

\author{
'Unidad de Gestión Clínica de \\ Medicina Preventiva, Vigilancia \\ y Promoción de la Salud. \\ Hospital Universitario San Cecilio \\ (Granada), España. \\ ${ }^{2}$ Servicio de Medicina Preventiva \\ y Salud Pública. Hospital \\ Universitario Central de Asturias. \\ Oviedo, España. \\ ${ }^{3}$ Departamento de Teoría y \\ Fundamentos de la Educación. \\ Universidad Internacional de la \\ Rioja (UNIR), España. \\ ${ }^{4}$ Centro de Investigaciones \\ Biomédicas en Red de Salud \\ Mental /CIBERSAM)-Universidad \\ de Granada, España. \\ ${ }^{5}$ Departamento Bioquímica y Biol. \\ Mol. III e Inmunol. Universidad de \\ Granada, España. \\ ${ }^{6}$ Instituto de Neurociencias \\ "Federico Olóriz". Universidad de \\ Granada, España.
}

Fuente de financiación y conflicto de intereses: Ninguna.

Recibido el 19 de junio de 2013, aceptado el 27 de enero de 2014.

Correspondencia a: María Fernández-Prada. Servicio de Medicina Preventiva. Hospital Universitario San Cecilio (Granada).

Avd. Dr. Olóriz, 18014.

Teléfono: (+34) 678120248

mariafdezprada@gmail.com

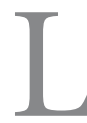

a calidad de vida relacionada con la salud (CVRS) es un constructo complejo para el que no hay una única definición acep$\operatorname{tada}^{1,2}$. Actualmente, la forma más estandarizada para su evaluación ha sido el cuestionario ShortForm Health Survey Questionnaire (SF-36 $\left.{ }^{\circledR}\right)^{3}$. Sus propiedades de medida han sido analizadas en numerosas publicaciones, además de contar con una versión española validada y baremada ${ }^{4-6}$. Este cuestionario resulta útil tanto en la evaluación de la percepción de salud de poblaciones generales como de subgrupos específicos ${ }^{7,8}$.

El personal sanitario ha sido estudiado en numerosas ocasiones de acuerdo con su entorno laboral y posibles efectos sobre su salud psicológi$\mathrm{Ca}^{9,10}$. Sin embargo, a diferencia de lo que ocurre en países vecinos ${ }^{11,12}$, hasta ahora en España muy pocos trabajos han abordado la CVRS en este grupo, siendo el médico interno residente la figura menos estudiada $^{12}$. En general, los datos indican que los sanitarios presentan una peor salud percibida que su población de referencia ${ }^{12}$ y registran una alta in- 
cidencia de depresión, ansiedad y, en algunos países, también suicidio ${ }^{13-16}$. Además, especialidades tales como cirugía, oncología, cuidados intensivos o urgencias, pueden favorecer situaciones con una alta carga emocional ${ }^{17,18}$. Así pues, el Servicio de Urgencias (SU) puede constituir, por sí solo, un entorno que propicie la aparición de problemas relacionados con su salud física y mental ${ }^{19,20}$. La elevada presión asistencial, las exigencias psicológicas a las que están sometidos, el bajo control sobre el puesto y la inseguridad laboral fruto de la situación económica actual, entre otros, hacen que los individuos sean más vulnerables a las alteraciones de su bienestar físico y psicológico ${ }^{19}$. Todo lo expuesto, puede agravarse en el caso de los médicos internos residentes (MIR) a quienes, a pesar de su corta experiencia laboral, se les otorga desde el primer momento una responsabilidad que, a menudo, no les corresponde ${ }^{20}$. Debido a esto, algunos autores les atribuyen una parte importante de los aspectos médico-legales que acontecen en el $\mathrm{SU}^{21}$, al mismo tiempo que otros demuestran que la etapa de formación médica es un factor importante para el desarrollo de trastornos psíquicos ${ }^{22}$.

Por otro lado, numerosos estudios han demostrado que existen diferencias de género en cuanto a la salud mental y los estados de ansiedad y depresión ${ }^{23}$. En este sentido, es frecuente encontrar en las mujeres resultados más desfavorables a nivel psicológico ${ }^{8,23}$. Esto es importante si se tiene en cuenta que, en los últimos años, la profesión médica está sufriendo una gran feminización y se espera que las cifras de mujeres médicos sigan aumentando en los próximos años ${ }^{24,25}$.

Por tanto, el objetivo de este trabajo ha sido analizar la calidad de vida relacionada con la salud en una muestra de MIR que realizan guardias en un SU, analizando las posibles diferencias entre mujeres y hombres.

\section{Método y sujetos}

\section{Tipo de estudio y población}

Se trata de un estudio descriptivo transversal. Los participantes fueron 71 médicos internos residentes del Hospital Universitario San Cecilio (Granada, España), 47 mujeres y 24 hombres, con una media de edad de $26,38 \pm 1,71$. Realizaban una media de $68,31 \pm 17,71$ horas mensuales en jornada de guardia en el Servicio de Urgencias. Hubo 30 residentes de primer año (R1), 22 residentes de segundo año (R2), 10 de tercero (R3) y 9 de cuarto (R4) de diferentes especialidades. El estudio se llevó a cabo entre los meses de febrero y abril de 2012, ambos inclusive. Los criterios de inclusión fueron los siguientes: (1) ser MIR y (2) realizar al menos 40 horas de guardia al mes en el SU. Se llevó a cabo un muestreo no probabilístico de tipo incidental con el fin de conseguir la mayor muestra posible. En el período de estudio, la plantilla de médicos internos residentes en el SU se componía de un total de 102 residentes (41 sujetos R1, 34 R2, 13 R3 y 14 R4), por lo que la tasa de participación en el estudio fue del $69,6 \%$.

\section{Herramienta de evaluación psicológica}

Se ha utilizado la versión española del cuestionario de salud SF-36 ${ }^{\circledR}$ (Short-Form Health Survey Questionnaire). Éste atiende a 8 dimensiones generales: 1) Función física: si la salud limita la realización de actividades diarias y cotidianas; 2 ) Rol físico: si la salud interfiere en el trabajo; 3) Dolor corporal; 4) Salud general; 5) Vitalidad: energía auto-percibida para hacer cosas; 6) Función social: grado en el que los problemas físicos o psicológicos afectan a la vida social; 7) Rol emocional: grado de interferencia de los problemas emocionales en la vida diaria y profesional; y 8) Salud mental: valoración general de estado de salud mental considerando aspectos como la depresión, la ansiedad, el autocontrol y el bienestar general. De acuerdo con lo anterior, las dimensiones físicas de la salud son: salud general, rol físico, dolor corporal y función física, y las dimensiones psicológicas son: vitalidad, función social, rol emocional y salud mental. El cuestionario se encuentra validado y baremado para la población española ${ }^{5}$.

\section{Procedimiento}

Se hicieron varias reuniones en las que se expusieron las líneas generales de la investigación, se proporcionó la hoja informativa y el consentimiento informado para la participación en estudio. Se solicitó que el cuestionario se respondiera en un solo momento temporal.

\section{Consideraciones éticas}

La colaboración fue voluntaria y desinteresada. Cada participante firmó un consentimiento informado en el que se incluyó la posibilidad de 
retirarse en cualquier fase del estudio. La investigación fue aprobada por el Comité de Ética de la Investigación Biomédica de Granada, Consejería de Salud, (España).

Análisis de los datos

Los análisis estadísticos se llevaron a cabo mediante el programa SPSS 15.0 (IBM()) y fueron los siguientes: (1) comprobación de la distribución normal de la muestra (estadístico de Shapiro-Wilks) y la homogeneidad de las varianzas (prueba Levene); (2) análisis de medidas de tendencia central y dispersión de la medida; (3) cálculo de las puntuaciones tipificadas para todas las variables donde se establecieron comparaciones o relaciones; 4) $t$ de Student para una muestra; (5) correlaciones bi-variadas de Pearson; (6) regresión lineal múltiple con el método "por pasos sucesivos" usando la probabilidad de F para un valor de entrada de 0,15 y de salida de 0,20 . Se consideró significativo un valor de $p$ inferior a 0,05 . Debemos matizar que todas las comparaciones para una muestra fueron realizadas con los valores de referencia para la población española (25-34 años) ${ }^{5}$.

\section{Resultados}

En la Tabla 1 se detallan las comparaciones realizadas entre las puntuaciones medias de cada dimensión del SF- $36^{\circledR}$ en función del sexo y la edad con respecto a sus valores poblacionales. Se aprecia cómo las mujeres se encuentran por debajo de los valores de referencia en cuatro de las ocho dimensiones del SF-36 ${ }^{\circledR}$, siendo especialmente relevantes la salud mental $(\mathrm{p}<0,05)$ y el funcionamiento social $(p<0,001)$. Por el contrario, los hombres están dentro del rango de los valores poblacionales de referencia en todos los casos.

Los resultados coinciden con estudios realizados en países europeos en los que las mujeres también puntúan más desfavorablemente que los hombres ${ }^{7,15,26}$.

En base a lo anterior, se analizan qué dimensiones predicen la salud mental y el funcionamiento social en las mujeres. Para la primera, la vitalidad es la dimensión que mejor lo hace $\left(\mathrm{r}^{2}=0,583\right.$; $\beta=0,772 ; \mathrm{t}=6,650 ; \mathrm{p}<0,001[0,457-0,860])$ mientras que para el funcionamiento social es la propia salud mental la mejor predictora $\left(r^{2}=0,329 ; \beta=0,592 ; t=4,023 ; p<0,001[0,539\right.$ $1,65])$.

Estos datos pueden relacionarse con los descritos por otros autores que indican una mayor prevalencia de depresión y ansiedad en mujeres que hombres ${ }^{13,23,26}$, la influencia del sexo en la predicción de la depresión ${ }^{27}$, una elevada prevalencia de comorbilidades psiquiátricas entre los médicos ${ }^{22,24}$ y unos niveles subóptimos de calidad de vida percibida en sanitarios en formación ${ }^{22,28-30}$. Un estudio con personal sanitario español refiere también que este colectivo se encuentra por debajo de los valores obtenidos por su población de referencia, a excepción de las dimensiones funcionamiento físico y salud general ${ }^{31}$.

La Tabla 2 muestra las correlaciones de Pearson entre las distintas dimensiones del SF-36 ${ }^{\circledR}$ en función del sexo. Cabe destacar las diferencias

Tabla 1. Media, desviación típica y comparaciones de las puntuaciones del las ocho dimensiones del SF-36 ${ }^{\circledR}$ con sus valores poblaciones en varones $(n=24)$ y en mujeres $(n=47)$

\begin{tabular}{|c|c|c|c|c|c|c|}
\hline & $\begin{array}{l}\text { Media hombres } \\
\text { (desv. tip) }\end{array}$ & $\mathbf{T}$ & Sig. Bil & $\begin{array}{l}\text { Media mujeres } \\
\text { (desv.tip) }\end{array}$ & $\mathbf{T}$ & Sig. Bil \\
\hline Salud general & $79,00 \quad( \pm 12,42)$ & 0,102 & 0,921 & $76,87 \quad( \pm 16,30)$ & 0,165 & 0,870 \\
\hline Función física & $98,00 \quad( \pm 4,21)$ & 0,450 & 0,663 & $97,96 \quad( \pm 3,78)$ & 3,994 & 0,001 \\
\hline Rol físico & $95,00 \quad( \pm 15,81)$ & 0,240 & 0,816 & $82,03 \quad( \pm 34,91)$ & $-1,178$ & 0,248 \\
\hline Dolor corporal & $84,00 \quad( \pm 17,76)$ & $-0,961$ & 0,362 & $80,70 \quad( \pm 17,77)$ & $-1,081$ & 0,288 \\
\hline Vitalidad & $67,60 \quad( \pm 15,60)$ & $-1,480$ & 0,173 & $62,57 \quad( \pm 15,16)$ & $-2,844$ & 0,008 \\
\hline Funcionamiento social & $88,75 \quad( \pm 19,93)$ & $-1,166$ & 0,274 & $77,73 \quad( \pm 23,91)$ & $-3,635$ & 0,001 \\
\hline Rol emocional & $83,46 \quad( \pm 32,81)$ & $-1,179$ & 0,269 & $73,95 \quad( \pm 36,64)$ & $-2,584$ & 0,016 \\
\hline Salud mental & $75,70 \quad( \pm 15,52)$ & 0,596 & 0,596 & $70,00 \quad( \pm 12,93)$ & $-1,925$ & 0,050 \\
\hline
\end{tabular}

Desv.Típ $=$ Desviación típica; $t=$ Valor de T para una muestra. Sig.Bil= Significación bilateral para una $p<0,05$. 
Calidad de vida en médicos residentes que hacen guardias en Urgencias - M. Fernández-Prada et al

Tabla 2. Correlaciones bivariadas de Pearson entre las distintas dimensiones del SF-36 ${ }^{\circledR}$ para hombres $(n=24)$ y mujeres $(n=47)$

\begin{tabular}{|c|c|c|c|c|c|c|c|}
\hline & $\begin{array}{l}\text { Función } \\
\text { física }\end{array}$ & Rol físico & Vitalidad & $\begin{array}{l}\text { Dolor } \\
\text { corporal }\end{array}$ & $\begin{array}{l}\text { Función } \\
\text { social }\end{array}$ & $\begin{array}{c}\text { Rol } \\
\text { emocional }\end{array}$ & $\begin{array}{l}\text { Salud } \\
\text { mental }\end{array}$ \\
\hline Salud general & $\begin{array}{l}0,064^{1} \\
0,077^{2}\end{array}$ & $\begin{array}{l}0,254 \\
0,337\end{array}$ & $\begin{array}{l}0,261 \\
0,417^{*}\end{array}$ & $\begin{array}{l}0,196 \\
0,494^{* *}\end{array}$ & $\begin{array}{l}0,258 \\
0,224\end{array}$ & $\begin{array}{l}0,136 \\
0,273\end{array}$ & $\begin{array}{l}0,283 \\
0,346\end{array}$ \\
\hline Función física & & $\begin{array}{l}0,667^{* 1} \\
0,255^{2}\end{array}$ & $\begin{array}{l}0,797^{* *} \\
0,211\end{array}$ & $\begin{array}{l}0,564 \\
0,172\end{array}$ & $\begin{array}{l}0,859^{* *} \\
0,197\end{array}$ & $\begin{array}{l}0,814^{* *} \\
0,072\end{array}$ & $\begin{array}{l}0,855^{* *} \\
0,139\end{array}$ \\
\hline Rol físico & & & $\begin{array}{l}0,351^{1} \\
0,503^{* * 2}\end{array}$ & $\begin{array}{l}0,870^{* *} \\
0,349\end{array}$ & $\begin{array}{l}0,903^{* *} \\
0,447^{*}\end{array}$ & $\begin{array}{l}0,901 * * \\
0,043\end{array}$ & $\begin{array}{l}0,706^{*} \\
0,337\end{array}$ \\
\hline Vitalidad & & & & $\begin{array}{l}0,407^{1} \\
0,412^{* 2}\end{array}$ & $\begin{array}{l}0,663^{*} \\
0,564^{* *}\end{array}$ & $\begin{array}{l}0,603 \\
0,440 *\end{array}$ & $\begin{array}{l}0,872^{* *} \\
0,772^{* *}\end{array}$ \\
\hline Dolor corporal & & & & & $\begin{array}{l}0,808 * * 1 \\
0,410 * 2\end{array}$ & $\begin{array}{l}0,900^{* *} \\
0,376^{*}\end{array}$ & $\begin{array}{l}0,625 \\
0,223\end{array}$ \\
\hline Función social & & & & & & $\begin{array}{l}0,967 * * 1 \\
0,529 * * 2\end{array}$ & $\begin{array}{l}0,865^{* *} \\
0,592^{* *}\end{array}$ \\
\hline Rol emocional & & & & & & & $\begin{array}{l}0,795^{* * 1} \\
0,540^{* * 2}\end{array}$ \\
\hline
\end{tabular}

1 = la fila superior muestra los índices de correlación para varones; 2 = la fila inferior muestra los índices de correlación para mujeres; ** $(p<0,001) ;{ }^{*}(p<0,050)$.

existentes entre hombres y mujeres, especialmente en relación con las dimensiones físicas y la salud mental. Las mujeres presentan un número menor de dimensiones que se relacionan positivamente con la salud mental. Esto se encuentra en consonancia con el efecto negativo del sexo sobre la CVRS, aspecto que fue puesto de manifiesto en un estudio nacional en $1998^{6}$.

Debemos resaltar lo anterior en el contexto de la creciente feminización de la profesión médi$\mathrm{ca}^{24,25} \mathrm{y}$, por tanto, la potencial afectación de una mayoría de los profesionales que deben velar por la salud de la población.

A nivel operativo, las posibles implicaciones prácticas de estos resultados deberían centrarse en conocer la percepción de la CVRS en los profesionales sanitarios con el fin de poner en marcha estrategias preventivas y/o de actuación que mejoren la situación física y/o psicológica de este colectivo. Una adecuada atención a este respecto puede redundar en beneficios directos para los propios trabajadores y la atención a los pacientes. Además, de forma más general, se reducirían incidencias, bajas laborales, y otros aspectos de interés para el sistema sanitario que pudieran derivarse de una situación aguda o prolongada de estos problemas $^{14-15}$. A nivel de gestión y coordinación de los SU, estos datos también pueden suponer una ayuda a los responsables a la hora de establecer acciones en sus áreas.

Debemos tener en cuenta que el trabajo presenta algunas limitaciones. La tasa de participación cercana al $70 \%$ podría afectar la calidad de los resultados, aunque el grupo de no participantes comparte una distribución parecida en cuanto al sexo y edad. En contraposición, hay que resaltar la homogeneidad de la muestra como una fortaleza y matizar que este problema suele ser común en diversos estudios sobre esta temática ${ }^{13,32}$. Si bien se ha realizado un estudio unicéntrico, las características de nuestro hospital son similares a las de otros de segundo nivel en cuanto a la organización del SU, el número de residentes por año y el perfil de la población que asiste. Esto se traduce en que los resultados de nuestro estudio podrían ser parcialmente compartidos con otros entornos. En futuros trabajos, sería interesante abordar esta realidad desde un estudio multicéntrico y relacionar la CVRS con otros constructos, tales como el burnout o los riesgos psicosociales en el trabajo con el fin de plantear estrategias preventivas que aborden la problemática desde un punto de vista biopsicosocial.

Para finalizar y conectando con el objetivo del 
estudio, las conclusiones más relevantes son que las mujeres MIR que realizan guardias en el SU tienen una percepción significativamente peor de su CVRS que la población general de referencia para su edad y sexo, especialmente en las dimensiones salud mental y funcionamiento social.

\section{Referencias}

1. Foster JH. Quality of life measurement and alcoholism: another arm to nursing practice? Clin Effect Nurs. 2006; 9: 259-301.

2. Urzúa MA. Calidad de vida relacionada con la salud: elementos conceptuales. Rev Med Chile 2010; 138: 358365.

3. Ware JE, Sherbourne CD. The MOS 36-item short-form health survey (SF-36) (I). Conceptual framework and item selection. Med Care 1992; 30: 473-83.

4. Vilagut G, Ferrer M, Rajmil L, Rebollo P, PermanyerMiralda G, Quintana JM, et al. El cuestionario de salud SF-36 español: una década de experiencia y nuevos desarrollos. Gac Sanit 2005; 19: 135-50.

5. Garratt A, Schmidt L, Mackintosh A, Fitzpatrick R. Quality of life measurement: bibliographic study of patient assessed health outcome measures. BMJ 2002; 324: 141721.

6. Alonso J, Regidor E, Barrio G, Prieto L, Rodríguez C, de la Fuente L. Valores poblacionales de referencia de la versión española del Cuestionario de Salud SF-36. Med Clin (Barc) 1998; 111: 410-6.

7. Stavem K, Hofoss D, Aasland OG. Work characteristics and morbidity as predictors of self-perceived health status in Norwegian physicians. Scand J Public Health 2003; 31: 375-81.

8. Iglesias-García C, Prieto R. Calidad de vida en mujeres deprimidas mayores de 40 años. Actas Esp Psiquiatr 2012; 40: 221-7.

9. Tokuda Y, Hayano K, Ozaki M, Bito S, Yanai H, Koizumi $\mathrm{S}$. The interrelationships between working conditions, job satisfaction, burnout and mental health among hospital physicians in Japan: a path analysis. Industrial Health 2009; 47: 166-72.

10. Voltmer E. Schwappach DL. Frank E. Wirsching M. Spahn C. Work-related behavior and experience patterns and predictors of mental health in German physicians in medical practice. Family Medicine 2012; 42: 433-9.

11. Aasland OG, Olff M, Falkum E, Schweder T, Ursin H. Health complaints and job stress in Norwegian physicians: the use of an overlapping questionnaire design. Soc Sci Med 1997; 45: 1615-29.
12. Stavem K, Hofoss D, Aasland OG, Loge JH. The selfperceived health status of Norwegian physicians compared with a reference population and foreign physicians. Scand J Public Health 2001; 29: 194-9.

13. Failde I, Ramos I, Fernández-Palacín F, González-Pinto A. Women Mental Health and Health-Related Quality of Life in Coronary Patients. Women and Health 2006; 43: 35-49.

14. Iliceto P, Pompili M, Spencer-Thomas S, Ferracuti S, Erbuto D, Lester D, et al. Occupational stress and psychopathology in health professionals: An explorative study with the Multiple Indicators Multiple Causes (MIMIC) model approach. Stress 2013; 16: 143-52.

15. Aasland OG, Hem E, Haldorsen T, Ekeberg Ø. Mortality among Norwegian doctors 1960-2000. BMC Public Health 2011; 11: 173.

16. Austin AE, van den Heuvel C, Byard RW. Physician Suicide. J Forensic Sci 2013; 58: 91-93.

17. Yavuzsen T, Alacacioglu A, Dirioz M, Yilmaz U. Quality of life of physicians and nurses working in an oncology clinic. J Buon 2011; 16: 537-40.

18. González-Cabrera, Fernández-Prada M, Molina Ruano R, Blázquez A, Guillén Solvas J, Peinado JM. Riesgo psicosocial en el trabajo, estrés autopercibido y cortisol en saliva en una muestra de urgenciólogos de Granada. Emergencias 2012; 24: 101-6.

19. Escribà-Agüir V, Martín-Baena D, Pérez-Hoyos S. Psychosocial work environment and burnout among emergency medical and nursing staff. Int Arch Occup Environ Health 2006; 80: 127-33.

20. Fernández-Prada M, Hernández Cueto C. Riesgo y conflictividad médico-legal en los servicios de urgencias hospitalarios: valoración médico-forense [carta]. Emergencias 2013; 25: 236-7.

21. Giménez Pérez D, Galtés Vicente JI, Iglesias Lepine ML. Riesgo y conflictividad médico-legal en los servicios de urgencias hospitalarios: valoración médico-forense. Emergencias 2012; 24: 389-96.

22. Granada Jiménez O, Morales Socorro MP, López-Ibor Aliño JJ. Psicopatología y factores de riesgo durante la residencia. Actas Esp Psiquiatr 2010; 38: 65-71.

23. Rubio-Aranda E, Blasco-Montón G, Comín-Comín M, Martínez-Terrer T, Magallón-Botaya R, García-Campayo J. Salud mental y relaciones sociales en población mayor rural. Actas Esp Psiquiatr 2012; 40: 239-47.

24. Arrizabalaga P, Valls-Llobet C. Mujeres médicas: de la incorporación a la discriminación. Med Clin (Barc). 2005; 125: 103-7.

25. Arrizabalaga P, Bruguera M. Feminización y ejercicio de la medicina. Med Clin (Barc) 2009; 133: 184-6.

26. Coelho R, Martins A, Barros H. Clinical profiles relating 
gender and depressive symptoms among adolescents ascertained by Beck Depression Inventory II. Eur Psychiatry 2002; 17: 222-6.

27. Bellón JA, Luna J, King M, Moreno-Küstner B, Nazareth I, Montón-Franco, $\mathrm{C}$ et al. Predicting the onset of major depression in primary care: international validation of a risk prediction algorithm from Spain. Psychological Medicine 2011; 41: 2075-88.

28. Ramirez AJ, Graham J, Richards MA, et al. Mental health of hospital consultants: the effects of stress and satisfaction at work. Lancet 1996; 347: 724-8.

29. West CP, Shanafelt TD, Kolars JC. Quality of life, burnout, educational debt, and medical knowledge among internal medicine residents. JAMA 2011; 306: 952-60.

30. Rezende GL, Mello MS, Granjeiro RC, Nakanishi M, Oliveira CA. The quality of life among Otorhinolaryngology residents in Distrito Federal (Brazil). Brazilian Journal of Otorhinolaryngology 2011; 77: 466-72.

31. Suñer-Soler R, Grau-Martín A, Font-Mayolas S, Gras ME, Bertran C, Sullman MJ. Burnout and quality of life among Spanish healthcare personnel. J Psychiatr Ment Health Nurs 2013; 20: 305-13.

32. Matía AC, Cordero J, Mediavilla J, Perea Riguera MJ. Prevalencia de burnout y variables asociadas en los médicos de atención primaria de Burgos. Aten Primaria 2006; 38 : 58-9. 